\section{Gestores do SUS: apoio e resistências à Homeopatia}

\author{
Support for and resistance to Homeopathy among \\ managers of the Unified National Health System
}

\author{
${ }^{1}$ Faculdade de Medicina, \\ Universidade de São Paulo, \\ São Paulo, Brasil. \\ Correspondência \\ S. A. C. Salles \\ Departamento de Medicina \\ Preventiva, Faculdade de \\ Medicina, Universidade de \\ São Paulo. \\ Rua Tomé de Souza 130, São \\ Paulo, SP 05079-000, Brasil. \\ sandrachaim@terra.com.br
}

\section{Abstract}

This article presents partial findings from a study on trends towards greater or lesser proximity between homeopathic and allopathic physicians, from the perspective of the latter. Forty-eight health professionals were interviewed (faculty, managers, and physicians working in the public health system). This specific article focused only on the interviews with health system managers. The following concepts were used as references: social and scientific field (Bourdieu); medical rationalities (Madel Luz); technological arrangements in health work (Mendes-Gonçalves); and physician's professional identity (Donnangelo \& Schraiber). According to the findings, support by managers for the presence of Homeopathy in the Unified National Health System is related to their perception of social demand, defense of patients' right to choose, and the observation that it is a medical practice that reclaims the humanist dimension of medicine, thus contributing to user satisfaction. The difficulties and resistances identified by managers highlight that the lack of information on homeopathic procedures limits the possibilities for use of Homeopathy because it leads to insecurity towards this area of medicine.

Homeopathy; Medicine; Public Health
Sandra Abrahão Chaim Salles 1 Lilia Blima Schraiber 1

\section{Introdução}

Este artigo propõe algumas reflexões sobre a assistência médica homeopática no Sistema Único de Saúde (SUS) com base em parte dos resultados de pesquisa conduzida entre os anos de 2002 e 2005.

O objetivo principal era investigar as características presentes na relação que se estabelece nos dias atuais entre a Homeopatia e a Biomedicina, segundo o ponto de vista dos médicos não homeopatas.

A Homeopatia é um sistema médico complexo. Diferencia-se da medicina ocidental contemporânea (Biomedicina) em seu sistema diagnóstico e de intervenção terapêutica, que opera segundo concepções próprias sobre a morfologia humana, dinâmica vital e doutrina médica 1 . Não deve ser reduzida, portanto, a um recurso terapêutico. Apesar de ser uma outra racionalidade médica, ela vem sendo gradativamente incorporada às instituições de saúde do nosso país, sejam elas de assistência, pesquisa ou ensino. Esse processo de institucionalização teve início a partir do seu reconhecimento como especialidade médica, pelo Conselho Federal de Medicina, em 1980, que, por sua vez foi uma decorrência de sua legitimação social.

Na saúde pública os movimentos iniciais de inclusão da Homeopatia foram iniciativas individuais de alguns médicos que tinham formação em homeopatia e obtiveram permissão para 
atender pacientes, como homeopatas, em agenda paralela. Mas foi a ação coletiva de grupos de homeopatas desenvolvendo um trabalho político e técnico em defesa da Homeopatia na saúde pública que resultou em um movimento de institucionalização de fato da Homeopatia, com a progressiva inserção do atendimento no planejamento dos serviços e nas políticas de saúde.

Atualmente, a Homeopatia é uma opção para os usuários do SUS, uma vez que é oferecida pela rede ambulatorial de cerca de 108 municípios. Mas, a falta de uma política ministerial para o desenvolvimento da Homeopatia no SUS ainda repercute no campo e uma das evidências desta condição atípica da Homeopatia, reconhecida como especialidade médica e farmacêutica, mas não contemplada pelas políticas publicas, é a falta de acesso dos usuários ao medicamento homeopático. A aprovação e publicação da Política Nacional de Práticas Integrativas e Complementares no Sistema Único de Saúde, em maio de 2006 pelo Ministério da Saúde, poderá modificar essa situação, uma vez que propõe uma política nacional para a assistência homeopática.

Nas faculdades de medicina, a Homeopatia ainda é pouco presente, dependendo sempre da iniciativa dos homeopatas, que têm buscado estas instituições como espaços de ensino e pesquisa, contribuindo para incrementar a produção e a publicação de trabalhos sobre o saber e a prática homeopáticos.

Nesses dois ambientes, Academia e SUS, a convivência entre essas duas medicinas é uma realidade que se impõe, seja por meio do contato direto entre os profissionais, ou indiretamente por intermédio dos pacientes usuários das duas medicinas.

A contínua ampliação do uso da Homeopatia e outras formas de cuidado, diferentes da Biomedicina, têm sido observadas em quase todo o mundo, o que tem despertado o interesse de muitos pesquisadores em conhecer melhor esse processo. Alguns artigos têm sido publicados com reflexões sobre a percepção de profissionais de saúde (médicos ou não) a respeito de um conjunto de práticas em saúde diferente da medicina hegemônica, denominadas medicinas alternativas, complementares ou não convencionais, que englobam a Homeopatia, Acupuntura, Naturopatia, Fitoterapia, práticas manipulativas como Osteopatia e Reflexologia, entre outras. Ainda que mereçam ressalvas, por avaliarem um conjunto que inclui especialidades médicas reconhecidas no Brasil (Homeopatia e Acupuntura) e práticas terapêuticas com diferentes níveis de regulação, uma conclusão comum aos diferentes trabalhos merece ser citada pois aponta para a realidade do pluralismo terapêutico no campo da saúde e a conseqüente necessidade de ampliar a divulgação de informações a respeito destas práticas entre os profissionais de saúde 2,3,4,5,6.

Os resultados aqui apresentados falam das características dessa aproximação que vem sendo observada no campo da saúde em nosso país, onde agentes/sujeitos de seus respectivos saberes interagem, como em todo universo social, em tensão, refletindo conflitos e convergências históricos. Este artigo vai se restringir a apresentar um dos elementos investigados - a relação dos gestores com a presença da Homeopatia no SUS, buscando analisar aspectos relevantes envolvidos no seu apoio a este movimento, assim como as resistências que eles observam e descrevem em suas entrevistas.

\section{Desenho da pesquisa e referenciais usados}

A investigação como um todo foi realizada a partir de entrevistas em profundidade com profissionais de saúde não-homeopatas, docentes e pesquisadores, gestores e médicos que atendem no SUS, de diferentes municípios e faculdades de medicina do país. Foram escolhidos, para as entrevistas, 16 gestores dos seis municípios que tiveram a maior produção ambulatorial de consultas em Homeopatia no ano de 2003 (Departamento de Informática do SUS. http://www. datasus.gov.br): Rio de Janeiro, São Paulo, Vitória (Espírito Santo), Juiz de Fora (Minas Gerais), Dourados (Mato Grosso do Sul) e Brasília (Distrito Federal).

Esses gestores possuem diferentes graus de envolvimento com a Homeopatia - enquanto alguns apenas administram uma diretoria regional de saúde que abriga um serviço de Homeopatia, outros participaram ativamente de projetos de implantação da assistência homeopática no município. Dessa forma, a amostra contemplou alguns simpatizantes da Homeopatia e também outros, que são indiferentes ou não acreditam nesta proposta, mas que são instados, no exercício de suas funções, a conviver com ela. Todos eles foram convidados a refletir sobre a presença da Homeopatia no SUS.

Neste estudo foram usadas, de maneira complementar, as concepções de Bourdieu a respeito de campos sociais e as reflexões de Maria Cecilia Donnangelo, apresentando as diferentes ideologias sustentadas pelos médicos, tendo como sistema de referência a sua condição de categoria profissional homogênea e também de trabalhadores especializados ocupando posições no conjunto do sistema de produção de serviços. Os estudos sobre a identidade de médico descrevendo 
os conflitos que surgem nas dimensões éticas da profissão, com base na sua inscrição no mercado de trabalho 7, também foram referências importantes para a análise dos resultados.

O projeto de pesquisa foi submetido e aprovado pela Comissão de Ética para Análise de Projetos de Pesquisa do Hospital das Clínicas e Faculdade de Medicina, Universidade de São Paulo (USP), e todos os participantes assinaram o Termo de Consentimento Livre e Esclarecido.

\section{Resultados}

Os resultados das entrevistas com os gestores abrangem vários temas ligados às grandes questões da saúde pública e do ensino médico, revelando os valores nos quais baseiam seus argumentos em defesa da Homeopatia no SUS ou suas resistências à aceitação plena desta medicina como uma opção para a população. Serão apresentados a seguir os principais núcleos temáticos identificados como argumentos de apoio ou resistência dos gestores à Homeopatia, com as citações dos entrevistados em itálico identificadas apenas pelo cargo que já exerceram.

\section{Possibilidade de escolher:}

direito de cidadania

"Então, o quê que na verdade eu vislumbrava? A possibilidade de universalização do seu acesso. Quer dizer, aqueles que desejam ser usuários dessa prática, eu gostaria muito de poder estar disponibilizando universalmente, da mesma forma que uma prática alopática" (Coordenador de atenção primária, Secretaria Estadual de Saúde, ex-secretário de saúde).

"Promover a eqüidade. Ela estava restrita aos pacientes abonados que poderiam pagar uma consulta na rede privada. Nada mais justo então que facilitar o acesso à Homeopatia e Acupuntura para todos" (Subsecretário de assistência à saúde, Secretaria Estadual de Saúde).

“...Eu tinha essas coisas do SUS como quase um mote existencial, então aqueles princípios da universalidade de acesso, de que o cidadão tem direito, isso tudo me motivava muito porque era uma ferramenta a mais... o que nos motivou muito era que nós tínhamos uma leitura política muito aguçada da questão do direito e do acesso. Então aquilo era uma coisa que já era reconhecida pelo Conselho Federal de Medicina, havia uma expectativa de usuários, havia um grupo de médicos que queria trabalhar com isso e nós entendíamos que era direito do povo, aquela coisa muito politizada mesmo, então isso ajudou muito a gente a viabilizar o serviço" (Ex-secretário municipal de saúde).
Os gestores reconhecem a demanda social como força de pressão política para promover mudanças dentro do sistema, sugerindo que sua ação para universalizar o acesso à Homeopatia se baseia em razões de natureza administrativa e também política. Perceber a demanda social e a impossibilidade de acesso pela escassez de médicos homeopatas foi fator motivador para atitudes de apoio dos gestores entrevistados às iniciativas para a ampliação dos serviços de Homeopatia na rede. Nesse momento eles tomam para si a função de, enquanto gestores da assistência pública à saúde, promover a justiça social por meio da universalização do acesso a uma medicina que é reconhecida como especialidade médica. Esse argumento é relevante, pois foi apresentado por gestores de todos os municípios visitados e já havia sido utilizado em outros estudos, como o de Novaes ${ }^{8}$ (p. 93) ao considerar que "a possibilidade de se estender à população, pelo menos à parcela que acredita e busca, uma determinada terapêutica, constitui-se em direito de cidadania".

\section{O olhar administrativo do gestor}

"Não porque era um bando de gente santinha, era muito uma exigência da prática mesmo, a questão filosófica - de escutar a pessoa, de vê-lo como um todo, que exigia uma consulta maior, que gerava um acolhimento melhor e confortava as pessoas. Isso do ponto de vista da gestão publica, das nossas dificuldades no SUS que tem um grande problema de respeito ao cidadão, de acolhimento, de consultas de baixa qualidade, isso foi muito motivador" (Ex-secretário municipal de saúde).

“...Eu tenho que te confessar, o meu olhar é o do gerente de serviço, do conjunto de serviços. A Homeopatia tem uma adesão fantástica dos usuários - quem entra não quer sair do serviço. Mais de 95\% de adesão. O serviço de Homeopatia requer muito pouco aparato de apoio de diagnóstico. Então, dentro da minha racionalidade, eu imagino o seguinte: se a gente tem um modelo de prática médica, a medicina, que tem se mostrado incapaz, insuficiente de garantir integralidade, universalidade, igualdade de acesso e satisfação do usuário, nós temos um outro modelo, outros modelos de prática que podem, sem ser excludentes, igual a medicina foi ao longo das décadas com as terapêuticas não convencionais, mas estar sendo uma opção terapêutica do usuário, por que não implementá-lo?" (Coordenador de planejamento, ex-superintendente de assistência à saúde - Secretaria Municipal de Saúde).

“...Entre consultas, exames complementares e medicação, o paciente sai muito mais barato do que um paciente que é atendido na alopatia. Isso 
na questão financeira. Na questão do paciente, eu acho que a questão, vendo agora lado do paciente, do usuário do SUS, ele vai ter um atendimento muito mais humanitário: sem aquela correria do alopata que atende ali e em poucos minutos, já pedindo vários exames... o homeopata, por tocaro paciente, por atender e gastar um tempo necessário pro atendimento, ele fica todo já coberto, então qualquer procedimento que ele faz ele está totalmente embasado daquilo que ele está fazendo" (Gerente de atenção secundária).

Os gestores são sensíveis à avaliação da clientela de serviços homeopáticos sobre a qualidade do atendimento e ao assumir a defesa do direito de acesso a toda a população que demandar a Homeopatia, esses entrevistados expressam a ideologia ocupacional do gestor, que tem na promoção da satisfação do usuário uma das metas de sua função no sistema de saúde.

Ao apontar, como justificativa de apoio à Homeopatia, o seu baixo custo, levantam a suspeição de estarem em defesa de uma medicina barata que poderia possibilitar a ampliação da cobertura da atenção primária. Mas desfazem essa impressão apresentando esse argumento de forma vinculada à qualidade do cuidado homeopático, descrevendo-o como um encontro entre médico e paciente que se realiza segundo os padrões éticos da boa prática médica.

Os entrevistados demonstram reconhecer que a consulta homeopática envolve uma anamnese extensa, acompanhada de um exame detalhado de cada paciente e que estes passos são necessários para que o homeopata efetive sua terapêutica - não se trata, portanto, de uma característica pessoal do homeopata, mas sim de uma exigência técnica da racionalidade em que ele opera, pois o homeopata assume para si o papel de reflexão crítica sobre o diagnóstico, freqüentemente delegado à tecnologia pela Biomedicina. Quando descrevem o ato médico homeopático como um ato dirigido e assegurado pela escuta e observação dos pacientes, os gestores estão reafirmando um valor universal para a prática médica, ou seja, descrevem o que seria a boa prática. Ao descrever a prática médica não-homeopática, em contraposição, referem-se ao excesso de pedidos de exames e às consultas rápidas. As representações sobre uma e outra medicina são claramente expressas por esses gestores e merecem uma atenção reflexiva, uma vez que "cada indivíduo é portador da cultura e das subculturas às quais pertence e é representativo delas" 9 (p. 194).

Ao buscar se institucionalizar, a Homeopatia o fez defendendo alguns elementos de sua prática considerados essenciais para garantir a eficácia de suas ações. O tempo de consulta foi um dos principais aspectos negociados nesse processo. Simultaneamente em que os homeopatas que atuavam na rede buscavam se adaptar a um tempo de consulta menor do que aquele que era proposto por algumas escolas de Homeopatia, sem comprometer a qualidade de sua prática 10 , os seus representantes demandavam a regulamentação do direito de consultas de uma hora (primeira vez) ou trinta minutos (retornos). Essa prerrogativa para sua prática, justificada nas características do seu saber, permitiu aos homeopatas a preservação da consulta como espaço relevante para o exercício da medicina. Ao fazêlo, a Homeopatia se diferencia das demais especialidades da medicina que, por valorizarem a tecnologia como uma garantia da cientificidade dos seus atos, foram, aos poucos, construindo o atual arranjo tecnológico em saúde, em que a consulta representa parcela de menor valor, simbólico e econômico.

Portanto, não podemos dizer que apenas o homeopata é capaz de uma prática médica que resgata a dimensão humanista da medicina, pois todos os médicos são formados como profissionais com uma ideologia que preserva este valor. Mas, podemos afirmar que as escolhas da medicina, em seu caminho de aproximação com a ciência, construíram para ela um modelo de assistência que dificulta esse resgate. E os gestores utilizam suas observações sobre a Homeopatia para explicitar críticas a esse modelo, ou desvio de modelo, como propõem alguns.

Os entrevistados também observam que a Homeopatia está adentrando em um sistema de saúde que a desconhece, pois foi a Biomedicina, como saber dominante, a construtora do modelo de saúde que hoje tenta abrigar a Homeopatia. Essa hegemonia se faz presente em todas as esferas do campo da saúde: política, científica, de divulgação e reprodução do saber, como o estudo procurou demonstrar. Eles observam ainda que mesmo após anos de discussões, críticas e reformas, esse modelo de atenção que aí está ainda valoriza muito a medicina hospitalar em detrimento das atividades ambulatoriais. Além disso, a lógica interna da prática na qual esse modelo se baseia, a Biomedicina, avaliza um conjunto de características - "o uso excessivo de exames complementares, a desvalorização da subjetividade do paciente e do próprio médico e a farmacologização excessiva" 11 (p. 135) - que se opõem frontalmente àquelas valorizadas pela prática homeopática.

Concluindo, podemos afirmar que a prática homeopática é caracterizada como uma ação que preserva a dimensão humanística da prática médica, pois defendeu, no processo de institucionalização, a preservação da consulta médica 
como ato de maior valor, pois sem isto não seria capaz de garantir a efetivação da sua atividade terapêutica.

\section{A Homeopatia como uma medicina do ser integral}

"Então, como eu tava te falando, por que a Homeopatia é maior? Por isso, porque a Alopatia, quanto mais se subespecializa, fica mais ligada mesmo à doença, aí trata dessa partezinha, enquanto ele vai no outro e trata do todo, ele não vai voltar em mim, então existe esse medo mesmo" (Gerente de atenção secundária).

"A cada dia que passa, onde a Alopatia não consegue dar conta do sujeito, e não consegue mesmo, do indivíduo como um todo... Você fala de outra coisa, você fala de um sujeito na íntegra, você fala de um sujeito que precisa de tempo, você fala de um sujeito que precisa ser observado com as suas conexões físicas e psíquicas. ...Acho que o problema maior que eu vejo é exatamente essa questão da integralidade que a medicina não dá conta, a oficial" (Coordenador de centro de referência de saúde do trabalhador).

"Eu acho que realmente tem questões da saúde que a população demanda que não se enquadram nos clássicos quadros clínicos da Alopatia, não se enquadram, e a gente não dá conta, enquanto que a Homeopatia consegue fazer, pela própria abordagem global, dá um atendimento que satisfaz mais a pessoa..." (Coordenador de centro de saúde escola).

Os gestores falam das dificuldades da Biomedicina, baseada na tecnologia das especialidades, em lidar com sintomas comumente trazidos pela população às unidades de saúde, por sua impossibilidade de compreender o indivíduo em sua totalidade. Apontam, ao mesmo tempo, como atributo distintivo, a Homeopatia como uma prática mais voltada para o sujeito integral.

Considerando que existem diversos sentidos de integralidade nos discursos que se apresentam no campo da saúde, é preciso saber reconhecer que os gestores entrevistados adotam, nessa avaliação que fazem das duas medicinas, um sentido de integralidade que se refere à medicina integral como aquela que não reduziria o paciente aos sistemas biológicos ou a suas queixas - em oposição à medicina reducionista e fragmentária apresentada pelos currículos das escolas médicas nos anos setenta. Ao apresentarem as suas experiências de ver a Homeopatia abordando os indivíduos doentes com essa compreensão e, paralelamente, constatarem a impossibilidade da Biomedicina de proceder da mesma forma, alguns profissionais entrevistados reapresentam um argumento que já foi usado por alguns estudiosos da saúde ao se colocarem em defesa do reconhecimento e incorporação de racionalidades médicas alternativas, pois as supunham mais amistosas para com a integralidade 12 .

Os gestores associam, ainda, a visão do todo do homeopata a uma possibilidade de atuar na promoção da saúde. Eles apresentam a idéia de que os conceitos de prevenção e promoção da saúde são mais compatíveis com práticas que trabalham o cuidado dos indivíduos dentro de um conceito positivo de construção da saúde como a Homeopatia, e não direcionadas para a doença, como a Biomedicina. Apontam ainda que essa mudança de visão que vem ocorrendo no campo da saúde pode aumentar a aceitação da prática homeopática. Alguns estudos já haviam sugerido que a Homeopatia, sendo uma prática que se propõe a cuidar do ser integral, numa perspectiva positiva de saúde, desenvolveria uma prática de promoção da saúde 13,14,15. Essa observação, vinda de gestores que tiveram contato com a Homeopatia em ação, na atenção básica, é importante testemunho que rebate aqueles que argumentam que a Homeopatia perderia, quando inscrita na rotina da saúde pública, suas características de prática humanista.

\section{As origens da resistência}

"Eu entendo que a Homeopatia rompe com a visão do biologicismo dentro da saúde. Ela rompe com o biologicismo e ela rompe exatamente com a fragmentação que existe dentro da abordagem. Então eu entendo a Homeopatia como uma especialidade médica, mas para além da medicina tradicional, da concepção médica hegemônica. ...Há todo esse confronto que é uma coisa muito comum quando você traz uma nova linguagem, quando você traz uma nova experiência e quando você rompe um pouco com essa questão que está colocada, com a linguagem habitual ... Vocêé uma ameaça. Você, da Homeopatia é uma ameaça à minha linha tradicional, biológica, centrada na doença onde o indivíduo não importa. Então o que você vai fazer? Os caras cerceiam mesmo. Acho que isso é o que eu tenho visto" (Coordenador de centro de referência à saúde do trabalhador).

"Olha, eu acho que é uma diferença tão grande, é uma questão epistemológica de princípio, de início: eu trato diversos doentes com o mesmo medicamento e a Homeopatia trata o doente com a sua doença, não sei nem se eles usam o termo doença. Então, o remédio é personalizado, eu acho muito difícil, há realmente uma distância muito grande até de leitura das coisas, a leitura do biológico, do funcionamento, então eu acho muito 
difícil a troca de figurinhas entre homeopatas e alopatas, porque eles têm lógicas muito distintas" (Ex-secretário municipal de saúde).

A capacidade de resolver problemas trazidos pelos pacientes é um valor essencial para a profissão ${ }^{16}$, mas a identidade profissional também é construída no compartilhamento de saberes sobre as doenças, tendo como base o conhecimento científico. E se o conhecimento científico confere aos participantes dessa profissão - os médicos - autoridade para legitimar um saber sobre as doenças, será a experiência clínica que lhes fundará o saber sobre doentes 17 . Por essa razão eles valorizam as práticas capazes de demonstrar resultados, mas a impossibilidade de compartilhar com elas os saberes herdados e reconhecidos como científicos vai lhes impedir de atribuir a elas a legitimidade científica que lhes garantiria o mesmo valor profissional no campo da saúde.

"Olha, eu acho que o que leva a maioria dos profissionais a não adotar a Homeopatia, ou não dar essa credibilidade à Homeopatia é o desconhecimento, vamos dizer assim, do princípio ativo e da ação do medicamento homeopático. ...Então eu acho assim, falta muita discussão em torno disso, falta esclarecimento para os profissionais" (Secretário municipal de saúde).

"Modelo que não tem as bases bioquímicas, biofísicas e fisiopatológicas que conhecemos. $E$ como todo doutor, pelo menos a sua maioria, o que ele não compreende, ele refuta, ele diz que não serve, ele simplesmente abomina; a não compreensão de ações energéticas, quer seja no desenvolvimento do processo terapêutico, quer seja no seu resgate, a não compreensão leva a refutá-lo. Quer dizer, eu não vou admitir que eu não entendo, eu não vou admitir que isso tá além daquilo que eu consigo compreender fisiologicamente, fisiopatologicamente, bioquimicamente, biofisicamente, isso tá além da minha compreensão, não foram essas bases que eu aprendi na minha formação, mas eu não posso dizer isso, então simplesmente eu refuto e digo que é uma prática que eu não aceito, porque eu prefiro a Alopatia" (Coordenador de atenção primária, Secretaria Estadual e Saúde, ex-secretário de saúde).

Eles descrevem acima o desenvolvimento da principal resistência de cunho cientifico à Homeopatia, que se baseia na não compreensão da ação das ultradiluições. Relatam que é difícil compreender uma medicina, como a Homeopatia, que não oferece a mesma explicação farmacológica para a ação das substâncias que utiliza. As ultradiluições há muito têm sido apontadas como o tendão de Achiles da Homeopatia pelas dificuldades na comprovação científica de sua ação. Muitos experimentos laboratoriais foram desenvolvidos para confirmar a essa ação
18,19,20,21, mas faltam explicações sobre os mecanismos e o local de sua ação.

“A primeira coisa que se diz é: 'isso é um quadro agudo, você não pode usar isso, a Homeopatia é para questões crônicas, arrastadas, processos outros... Eu acho que o que pega realmente é isso 'eu não acredito por isso, é muito lento, você toma muita coisinha, umas bolinhas, essas coisas todas e você não sabe bem para quê que é,' etc. Não é uma coisa direcionada, porque a primeira pergunta é 'esse aqui é para quê??'. Esse é para tratar asma, esse é para tratar não sei o quê, porque é a lógica da Alopatia" (Coordenador da atenção primária).

"Essa coisa da cura através da Homeopatia, a concepção dela é divergente de todo modelo que a gente tem histórico aí de mais de cem anos, das bactérias da vida. E você dizer que vai fazer um princípio ativo através de similares e diluições, isso tem um impacto. A construção dessa concepção na cabeça de um profissional que tradicionalmente lida com comprovação de drogas que vão lá e matam, né? Que é uma visão ainda que vem lá de trás, mas que ainda é muito forte na medicina, você mudou toda uma concepção teórica, mas é aquela concepção unicausal, foi um troço que foi lá e causou a doença no cidadão, eu tenho que ir lá e matar aquele negócio, fazer com que ele desapareça. A nossa cultura médica é muito forte em torno ainda do conceito da unicausalidade, voltada para o momento agudo da doença" (Subsecretário Estadual de Saúde).

Não compreendendo como age o medicamento homeopático, os entrevistados atribuem a ele uma ação geral e inespecífica, não direcionada a um determinado distúrbio, o que constrói uma imagem de que a Homeopatia seria uma medicina incapaz de dar respostas imediatas às doenças agudas e graves em contraposição à Biomedicina, que, em conformidade com a cultura atual, atende a exigência de resultados imediatos. A Biomedicina define, então, como padrão de boa prática que não basta resolver, é preciso resolver rapidamente. Para uma determinada queixa é preciso contrapor uma determinada substância que atue rapidamente fazendo-a desaparecer. Os entrevistados ressaltam que a formação dos médicos é voltada para o imediatismo, dificultando sua aceitação de uma medicina que teria uma outra mensuração do tempo: do tempo terapêutico, do tempo de adoecimento e do tempo de cura.

\section{Considerações finais}

Pode-se perceber que entre os entrevistados predomina a noção de Homeopatia como uma "medicina suave", que lentamente poderia promover 
a melhora dos sintomas. Alguns fatores contribuem para a construção dessa percepção e, um deles, certamente, é a falta de contato com resultados da Homeopatia em quadros agudos. Essa condição não se deve apenas à falta de estrutura dos serviços para atendimentos de casos agudos, mas também às dificuldades que ocorrem no Brasil na formação dos homeopatas, pois os cursos de especialização não oferecem aos seus alunos um treinamento em serviço para atendimento de casos agudos. Conseqüentemente, diante desses casos, grande parte dos formados em Homeopatia se sente insegura para utilizar apenas esta medicina e optam por associar recursos da Biomedicina 22. Essa atitude reafirma a noção de que a Homeopatia é insuficiente para lidar com situações mais graves ou que exijam respostas imediatas.

Soma-se a isso o fato de serem poucas as publicações de resultados de pesquisas com a utilização da Homeopatia em situações agudas, e, dentre as publicações, muitas serem realizadas com pequeno número de sujeitos e muitas vezes não seguirem o modelo exigido pela academia, ou seja, ensaio clínico duplo cego, placebo controlado 23,24,25,26,27,28 não sendo, portanto, suficientes para mudar a percepção dominante no campo da saúde de que a Homeopatia é indicada apenas nos casos de doenças crônicas.

As dificuldades e resistências apontadas pelos gestores ressaltam que a falta de informações esclarecedoras sobre os procedimentos homeopáticos limita as possibilidades de utilização da Homeopatia porque gera insegurança sobre esta medicina. Cabe aos homeopatas, portanto, nesse momento da interlocução com seus pares, promover reflexões sobre sua própria medicina, definindo conceitos e esclarecendo sua tecnologia, de forma a tornar públicas as características da sua boa prática, os limites de sua ação e as possibilidades de parcerias com outras ações de saúde.

\section{Resumo}

Este artigo apresenta parte dos resultados de pesquisa que investigou características do movimento de aproximação e afastamento entre homeopatas e médicos da Biomedicina, segundo o ponto de vista dos profissionais não homeopatas. Foram entrevistados 48 profissionais de saúde (docentes, gestores e médicos que trabalham na rede publica). Toma-se para análise apenas os resultados das entrevistas com gestores. Foram usadas como referências as concepções de: campo social e científico de Bourdieu; racionalidades médicas de Madel Luz; arranjos tecnológicos do trabalho em saúde de Mendes-Gonçalves e de identidade profissional de médico de Donnangelo e de Schraiber. Os resultados indicam que o apoio de gestores à presença da Homeopatia no SUS relaciona-se à percepção da demanda social, à defesa do direito de escolha dos usuários e à constatação de tratar-se de uma prática médica que resgata a dimensão humanista da medicina, contribuindo assim para a satisfação do usuário. As dificuldades e resistências apontadas pelos gestores ressaltam que a falta de informações sobre os procedimentos homeopáticos limita as possibilidades de utilização da Homeopatia porque gera insegurança sobre esta medicina.

Homeopatia; Medicina; Saúde Pública

\section{Colaboradores}

O artigo foi escrito por S. A. C. Salles com a colaboração e revisão de L. B. Schraiber.

\section{Agradecimentos}

À Fundação de Amparo à Pesquisa do Estado de São Paulo pelo apoio (processo no. 04/05973-8) e à Coordenação de Aperfeiçoamento de Pessoal de Nível Superior pela bolsa de Doutorado concedida à S. A. C. Salles. 


\section{Referências}

1. Luz MT. Comparação de representações de corpo, saúde, doença e tratamento em pacientes e terapeutas de homeopatia, acupuntura e biomedicina. Rio de Janeiro: Instituto de Medicina Social, Universidade do Estado do Rio de Janeiro; 1998. (Série Estudos em Saúde Coletiva).

2. Verhoef M, Sutherland LR. General practioners' assessment of and interest in alternative medicine in Canada. Soc Sci Med 1995; 41:511-5.

3. Tovey P. Contigent legitimacy: UK Alternative practioners and inter-sectorial acceptance. Soc Sci Med 1997; 45:1129-33.

4. Homar JC. ¿Medicinas complementarias o alternativas? A dilemma for the public health system? Aten Primaria 2005; 35:389-91.

5. Perdiguero E. El fenomeno del pluralismo asistencial: una realidad por investigar. Gac Sanit 2004; 18:140-5.

6. Anunciação EM, Lewisky P. Serviço municipal de homeopatia. Divulg Saúde Debate 2000; 19:88-90.

7. Schraiber LB. No encontro da técnica com a ética: o exercício de julgar e decidir no cotidiano do trabalho em Medicina. Interface Comun Saúde Educ 1997; 1:123-40.

8. Novaes RL. Sobre a homeopatia. Saúde Debate 1988; 22:85-94.

9. Michelat G. Sobre a utilização da entrevista nãodiretiva em sociologia. In: Thiollent M, organizador. Crítica metodológica, investigação social e enquete operária. São Paulo: Editora Polis; 1981. p 91-211.

10. Salles SAC. O perfil do médico homeopata [Dissertação de Mestrado]. São Paulo: Faculdade de Saúde Pública, Universidade de São Paulo; 2001.

11. Camargo Jr. KR. Biomedicina saber \& ciência: uma abordagem crítica. São Paulo: Editora Hucitec; 2003.

12. Mattos R. Os sentidos da integralidade: algumas questões acerca de valores que merecem ser defendidos. In: Pinheiro R, Mattos RA, organizadores. Os sentidos da integralidade na atenção e no cuidado à saúde. Rio de Janeiro: ABRASCO; 2001.

13. Galvão GG. Outros modelos de atenção à saúde: a medicina homeopática na Rede Pública [Dissertação de Mestrado]. Rio de Janeiro: Instituto de Medicina Social, Universidade do Estado do Rio de Janeiro; 1999.

14. Costa A. Apoio social e a concepção do sujeito na sua integração entre corpo-mente: uma articulação de conceitos no campo da saúde pública [Dissertação de Mestrado]. Rio de Janeiro: Escola Nacional de Saúde Pública, Fundação Oswaldo Cruz; 2002.
15. Lacerda A, Valla V. Homeopatia e apoio social: repensando as práticas de integralidade na atenção e no cuidado à saúde. In: Pinheiro R, Mattos RA, organizadores. Construção da integralidade: cotidiano, saberes e prática em saúde. Rio de Janeiro: Instituto de Medicina Social, Universidade do Estado do Rio de Janeiro/ABRASCO; 2003.

16. Freidson E. Profession of medicine. A study of the sociology if applied knowledge. New York: Dodd Mead \& Company; 1972.

17. Schraiber LB. O médico e seu trabalho. Limites da liberdade. São Paulo: Editora Hucitec; 1993.

18. Gonçalves MI. O uso da homeopatia no tratamento da infecção urinária em ratas [Tese de Doutorado]. São Paulo: Faculdade de Medicina, Universidade Federal de São Paulo; 2001.

19. Belon P, Cumps J, Ennis M, Mannaioni PF, SainteLaudy J, Roberfroid M, et al. Inhibition of human basophil degranulation by successive histamine dilutions: results of a European multi-centre trial. Inflamm Res 1999; 48 Suppl 1:S17-8.

20. Belon P, Cumps J, Ennis M, Mannaioni PF, SainteLaudy J, Roberfroid M, et al. Histamine dilutions modulate basophil activation. Inflamm Res 2004; 53:181-8.

21. Bastide M, Lagache A. A communication process: a new paradigm applied do high-dilution effects on the living body. Altern Ther Health Med 1997; 3:35-9.

22. Salles SAC. O perfil dos médicos formados em homeopatia no Brasil, 1988-1998: fase I. Rev Homeopatia (AMHB) 2002; (4):12-9.

23. Leal SM. A prática homeopática na Unidade de Terapia Intensiva (UTI). Rev Homeopatia (São Paulo) 2000; 65:29-55.

24. Friese KH, Kruse S, Lundtke R, Moeller H. Homeopathic treatment of otitis media in children: comparison with conventional therapy. Int J Clin Pharmacol Ther 1997; 35:296-301.

25. Jacobs J, Springers DA, Crothers D. Homeopathic treatment in acute otitis media in children: a preliminary randomized placebo-controlled trial. Pediatr Infect Dis J 2001; 20:177-83.

26. Jacobs J, Jimenez M, Malthouse S, Chapman E, Crothers D, Masuk M, et al. Acute childhood diarrhoea - a replication. J Altern Complement Med 2000; 6:131-9.

27. Haddad MA. Homeopatia: medicina do sujeito - um caso de Ipeca. In: XXVII Congresso Brasileiro de Homeopatia. Brasília: Associação Médica Homeopática do Distrito Federal; 2004. p. 39.

28. Jacobs J. Treatment of acute childhood diarrhea with homeopathic medicine: a randomized clinical trial in Nicaragua. Pediatrics 1994; 93:719-25.

Recebido em 10/Mar/2008

Aprovado em 26/Mai/2008 